\title{
Molecular Signature for Lymphatic Invasion Associated with Survival of Epithelial Ovarian Cancer
}

\author{
E Sun Paik, MD \\ Hyun Jin Choi, MD \\ Tae-Joong Kim, MD, PhD \\ Jeong-Won Lee, MD, PhD \\ Byoung-Gie Kim, MD, PhD \\ Duk-Soo Bae, MD, PhD \\ Chel Hun Choi, MD, PhD
}

Department of Obstetrics and Gynecology, Samsung Medical Center, Sungkyunkwan University School of Medicine, Seoul, Korea
Correspondence: Chel Hun Choi, MD, PhD Department of Obstetrics and Gynecology, Samsung Medical Center, Sungkyunkwan University School of Medicine, 81 Irwon-ro, Gangnam-gu, Seoul 06351, Korea

Tel: $82-2-3410-3545$

Fax: 82-2-3410-0630

E-mail: chelhun.choi@samsung.com

Received February 28, 2017

Accepted May 9, 2017

Published Online May 22, 2017

\begin{abstract}
Purpose
We aimed to develop molecular classifier that can predict lymphatic invasion and their clin-
\end{abstract} ical significance in epithelial ovarian cancer (EOC) patients.

\section{Materials and Methods}

We analyzed gene expression (mRNA, methylated DNA) in data from The Cancer Genome Atlas. To identify molecular signatures for lymphatic invasion, we found differentially expressed genes. The performance of classifier was validated by receiver operating characteristics analysis, logistic regression, linear discriminant analysis (LDA), and support vector machine (SVM). We assessed prognostic role of classifier using random survival forest (RSF) model and pathway deregulation score (PDS). For external validation, we analyzed microarray data from 26 EOC samples of Samsung Medical Center and curatedOvarianData database.

\section{Results}

We identified 21 mRNAs, and seven methylated DNAs from primary EOC tissues that predicted lymphatic invasion and created prognostic models. The classifier predicted lymphatic invasion well, which was validated by logistic regression, LDA, and SVM algorithm (C-index of $0.90,0.71$, and 0.74 for mRNA and C-index of 0.64, 0.68, and 0.69 for DNA methylation). Using RSF model, incorporating molecular data with clinical variables improved prediction of progression-free survival compared with using only clinical variables $(p<0.001$ and $p=0.008$ ). Similarly, PDS enabled us to classify patients into high-risk and low-risk group, which resulted in survival difference in mRNA profiles (log-rank $p$-value $=0.011$ ). In external validation, gene signature was well correlated with prediction of lymphatic invasion and patients' survival.

\section{Conclusion}

Molecular signature model predicting lymphatic invasion was well performed and also associated with survival of EOC patients.

\section{Introduction}

Epithelial ovarian cancer (EOC) is one of the leading causes of death in patients with gynecologic malignancy and the incidence of EOC has been increasing gradually [1,2]. EOC presents at a late stage in most cases and is known to have high pathological and molecular heterogeneity [3,4]. Although $75 \%$ of EOC patients achieve complete response

\section{Key words}

Gene expression signature, Ovarian neoplasm, Human genome, Lymphatic metastasis 
unnecessary procedure should be avoided. Recently, a lot of effort was made to integrate molecular signatures for precise prognosis; this helps classify patients into disparate risk groups and may provide more personalized treatment [11]. However, information on predictors for lymphatic invasion status in EOC patients is still lacking, and few molecular prognostic classifiers are available.

The Cancer Genome Atlas (TCGA) offers considerable opportunities to increase our knowledge of the molecular foundation of cancer, and data at multiple molecular levels have recently became available [12]. Data from DNA methylation at the epigenetic level, which plays an important role in controlling gene activity, and from gene (mRNA) at the level of the transcriptome are the most representative datasets. As the cancer genome is rather complex and is associated with numerous molecular mechanisms [13,14], a single level of molecular data is insufficient to include all of the information associated with the process. By integrating multiple levels of molecular signatures for the layered processes of the biological system, a more acceptable prediction of the cancer phenotype can be provided.

In this study, we aimed to find predictors based on molecular signatures related to lymphatic invasion in EOC and to validate the prognostic significance of the signatures through analysis of mRNA, and methylated DNA expression profiles from the TCGA database.

\section{Materials and Methods}

\section{Patients and data collection for the prediction model}

Clinical information from TCGA Data Portal (https://tcgadata.nci.nih.gov/docs/publications/tcga/) and molecular data including DNA methylation, and mRNA were obtained before performing downstream analysis. Molecular data were used from the following platforms: for DNA methylation, the platform was Illumina Infinium Human DNA Methylation 27K (Illumina, San Diego, CA); for mRNA expression, the platform was Illumina HiSeq 2000 RNA Sequencing V2. This study meets the publication guidelines provided by TCGA (http:// cancergenome.nih.gov/ publications / publicationguidelines). Tables 1 and 2 show the data description of the multilevel genomic datasets for ovarian cancer, and patients' characteristics.

\section{Selection of differentially expressed genes according to lymphatic invasion}

To identify differentially expressed genes (DEG) between lymphatic invasion (-) and lymphatic invasion (+) tumors, level 3 pancancer-normalized expression levels were used. Because many genes were not expressed in certain samples or showed few variations between the patients in the dataset, only genes expressed in at least two lymphatic invasions (-) or lymphatic invasion $(+)$ samples were retained in the profile. The differences in expression were featured by $\log \mathrm{FC}$ (log2 fold change) and associated p-values. LogFC designates the fold change in expression of each gene from lymphatic invasion $(+)$ to lymphatic invasion (-). Downregulated and upregulated genes were distinguished as $\log \mathrm{FC}<-1$ and $\log \mathrm{FC}>1$, respectively, with false discovery rate (FDR)adjusted $\mathrm{p}<0.05$. To identify a molecular signature that is robustly associated with lymphatic invasion, we calculated the median FDR, which is the percentage of falsely detected genes as showing statistically significant DEG.

\section{Molecular signature for lymphatic invasion}

Logistic regression with backward elimination was applied to develop a prognostic model for lymphatic invasion using the selected genes. We used selected features to acquire a regression coefficient for each feature and to create a model to assess the prognosis prediction score as follows: prognostic score for $\mathrm{mRNA}=(2.3108+0.2983 \times \mathrm{HTR} 3 \mathrm{~A}-0.6302$ $\times$ PCP4-0.8574×CAPN9-0.7095×MASP1+1.3189×CYP8B1$0.4935 \times$ GDF5 $+1.7850 \times \mathrm{AOAH}+1.7531 \times$ ADORA3 $-0.4757 \times$ CLCNKB+0.7888 $\times$ EBI3 +1.0577 $\times$ LILRB3 $+1.9022 \times$ C16orf54+ $0.5381 \times$ PODNL $1+2.7914 \times \mathrm{DCN}+0.5553 \times \mathrm{USH} 2 \mathrm{~A}+2.1488 \times$ FGD2-5.9784×TNFAIP8L2-1.0032×SP140-3.1931×WISP1$0.7937 \times \mathrm{SH} 2 \mathrm{D} 4 \mathrm{~B}-1.9687 \times \mathrm{RUNX} 1)$; and prognostic score for methylation $=(-0.8776+1.9637 \times$ ITGBL $1+2.7273 \times$ CAPN9 + $2.6450 \times$ LOC $84391-4.3617 \times$ ACAP1-1.1581×ADORA3 $4.4722 \times \mathrm{FBN} 3+2.4902 \times \mathrm{CD} 37)$. The performance of the model was measured using receiver operating characteristics (ROC) curves by comparing the area under the curve (AUC) of the respective ROC.

\section{Validation of prediction accuracy}

Next, we compared the prediction efficiency of the logistic regression model with that obtained using the Logitboost model based on the same set of selected genes [15]. Internal validation was done by a 200 -fold cross-validation method to distinguish the samples as lymphatic invasion (-) or lymphatic invasion (+). This method to risk classification used the median index values acquired from $90 \%$ of the cases (training set) to divide the residual $10 \%$ of the missed cases (test set) according to this median estimate. All cases were stratified after the entire process had been repeated 200 times, with a different $10 \%$ of cases omitted until each case had been excluded. 
Table 1. Data description

\begin{tabular}{llc} 
Data type & \multicolumn{1}{c}{ Platform } & No. of features \\
Gene expression & HiSeqV2, Pancancer normalized & 20,530 \\
Methylation & Infinium human methylation 27 BeadChip & 27,578 \\
\hline
\end{tabular}

Table 2. Patients' characteristics of TCGA dataset

\begin{tabular}{|c|c|c|c|}
\hline Characteristic & $\begin{array}{l}\text { Lymphatic invasion (-) } \\
\qquad(\mathrm{n}=39)\end{array}$ & $\begin{array}{l}\text { Lymphatic invasion }(+) \\
\qquad(\mathrm{n}=70)\end{array}$ & p-value \\
\hline Age (yr) & $57(51.5-61.5)$ & $56.5(49-65)$ & 0.599 \\
\hline \multicolumn{4}{|l|}{ FIGO stage } \\
\hline IIA & 0 & $1(1.4)$ & 0.058 \\
\hline IIB & $1(2.6)$ & $1(1.4)$ & \\
\hline IIC & $3(7.7)$ & $6(8.6)$ & \\
\hline IIIA & $4(10.3)$ & 0 & \\
\hline IIIB & $4(10.3)$ & $2(2.9)$ & \\
\hline IIIC & $25(64.1)$ & $51(72.9)$ & \\
\hline IV & $2(5.1)$ & $9(12.9)$ & \\
\hline \multicolumn{4}{|l|}{ ECOG performance status } \\
\hline 0 & $4(44.4)$ & $13(76.5)$ & 0.122 \\
\hline 1 & $5(55.6)$ & $3(17.6)$ & \\
\hline 3 & 0 & $1(5.9)$ & \\
\hline \multicolumn{4}{|l|}{ Grade } \\
\hline G1 & 0 & $1(1.4)$ & 0.267 \\
\hline G2 & $7(17.9)$ & $5(7.1)$ & \\
\hline G3 & $32(82.1)$ & $63(90.0)$ & \\
\hline GX & 0 & $1(1.4)$ & \\
\hline \multicolumn{4}{|c|}{ Residual disease after primary treatment } \\
\hline Not available & $4(10.3)$ & $6(8.6)$ & 0.265 \\
\hline$>20 \mathrm{~mm}$ & $4(10.3)$ & $14(20.0)$ & \\
\hline $11-20 \mathrm{~mm}$ & $1(2.6)$ & $30(42.9)$ & \\
\hline $1-10 \mathrm{~mm}$ & $15(38.5)$ & $5(7.1)$ & \\
\hline No macroscopic disease & $15(38.5)$ & $15(21.4)$ & \\
\hline
\end{tabular}

Values are presented as median (range) or number (\%). Lymphatic invasion refers to as peri-tumoral lymphatic invasion. Residual disease after primary treatment was categorized by largest diameter described by surgeons in operation record. TCGA, The Cancer Genome Atlas; FIGO, International Federation of Gynecology and Obstetrics; ECOG, Eastern Cancer Oncology Group.

We further validated the classifier using two classification algorithms: linear discriminant analysis (LDA) and support vector machine (SVM). Both algorithms were repeated 100 times, and the accuracy was compared using the concordance index. Linear SVM classifier aims to find an optimal hyperplane to separate the classes in the high-dimensional gene space with the generalization ability maximized and over-fitting avoided [16]. We used the weighted difference of means of genes in the SVM decision function to evaluate the relative importance of genes and eliminated unimportant genes iteratively by a backward feature elimination process. SVM-based predictions function well on small sample sets in high-dimensional spaces [17].

\section{Molecular signature of lymphatic invasion predicted sur- vival of EOC patients}

We gathered candidate features of molecular data and randomized the data set into training $(80 \%)$ and test $(20 \%)$ sets. We then made the survival predictive models from the train- 
Table 3. Characteristics of 26 serous ovarian cancer patients from Samsung Medical Center

\begin{tabular}{|c|c|c|c|}
\hline Characteristic & $\begin{array}{l}\text { Lymphatic invasion (-) } \\
\qquad(\mathrm{n}=11)\end{array}$ & $\begin{array}{l}\text { Lymphatic invasion }(+) \\
\qquad(\mathrm{n}=15)\end{array}$ & p-value \\
\hline Age (yr) & $50.2 \pm 8.1$ & $53.5 \pm 12.1$ & 0.433 \\
\hline \multicolumn{4}{|l|}{ FIGO stage } \\
\hline IIIC & $9(81.8)$ & $11(77.3)$ & 0.971 \\
\hline IV & $2(18.2)$ & $4(26.7)$ & \\
\hline \multicolumn{4}{|l|}{ Grade } \\
\hline G1 & $1(9.1)$ & 0 & 0.267 \\
\hline G2 & $4(36.4)$ & $1(6.7)$ & \\
\hline G3 & $6(54.5)$ & $14(93.3)$ & \\
\hline \multicolumn{4}{|c|}{ Residual disease after primary treatment } \\
\hline Optimal $(<1 \mathrm{~cm})$ & $5(45.5)$ & $7(46.7)$ & $>0.999$ \\
\hline Suboptimal $(\geq 1 \mathrm{~cm})$ & $6(54.5)$ & $8(53.3)$ & \\
\hline
\end{tabular}

Values are presented as mean \pm standard deviation or number (\%). Lymphatic invasion refers to as peri-tumoral lymphatic invasion. Residual disease after primary treatment was categorized by largest diameter described by surgeons in operation record. FIGO, International Federation of Gynecology and Obstetrics.

ing set using random survival forest (RSF) [18]. The RSF models were created by the R package "RandomSurvivalForest" with the recommended default reference values. We then applied the models to the test set for prediction and assess the C-index. For each core set, the process mentioned above was reiterated 100 times to extract $100 \mathrm{C}$-index values. In comparing the performance between different data types, the results based on the Wilcoxon signed rank test to assess the p-value using 0.05 as the significance cutoff were used. To calculate the predictive power of incorporating molecular signatures with clinical factors, we adjusted the RSF method to contain both clinical variables (International Federation of Gynecology and Obstetrics [FIGO] stage, grade, lymphatic invasion, and residual disease status after primary treatment) and molecular features. We used the lymphatic invasion as the reference for creating the clinical RSF model. We then combined the molecular-level signatures more appropriate for the existing model by processing a feature-selection step against the remainders.

In addition, we used Pathifier (http:// www.weizmann.ac.il / pathifier), an algorithm that calculates pathway deregulation scores (PDS) for each sample based on expression data [19]. In brief, Hastie and Stuetzle's algorithm [20] was used to find a principle curve. Then, we project to the closest point from each sample, and the deregulation score of sample is defined as the distance along the curve. In this study, the reference set is composed of the sample without lymphatic invasion. The distance provides a measure of the extent to which the expression levels of the genes associated with lymphatic invasion were perturbed in each sample with lymphatic invasion. The algorithm transforms gene-level infor- mation into pathway-level information. Kaplan-Meier survival curves were drawn according to the PDS, and the logrank test $p$-values were calculated using the $R$ package "survival."

For external validation of the result, we used additional analysis of gene expression dataset from the study which we previously published [21]. Twenty-six serous adenocarcinomas with stage IIIC/IV were used, and 14 patients had recurrence within 6 months and the remaining 12 patients had no recurrences. Patients' characteristics are shown in Table 3. For further validation of the selected genes, we used a meta-analysis using gene expression data on tumors, with carefully curated clinical annotations, which is available in the curatedOvarianData database [22].

\section{Statistical analysis}

In each data set, we used Monte Carlo cross-validation and calculated the predictive power of individual molecular data types using the $\mathrm{C}$-index [23]. The $\mathrm{C}$-index is a nonparametric indicator to quantify the differential power of a prediction model: a C-index of 0.5 is considered as good as a random guess. Progression-free survival (PFS) and overall survival (OS) curves were created with the Kaplan-Meier method and the log-rank test. We conducted all statistical analyses with the R statistical language ver. 3.1.0. 


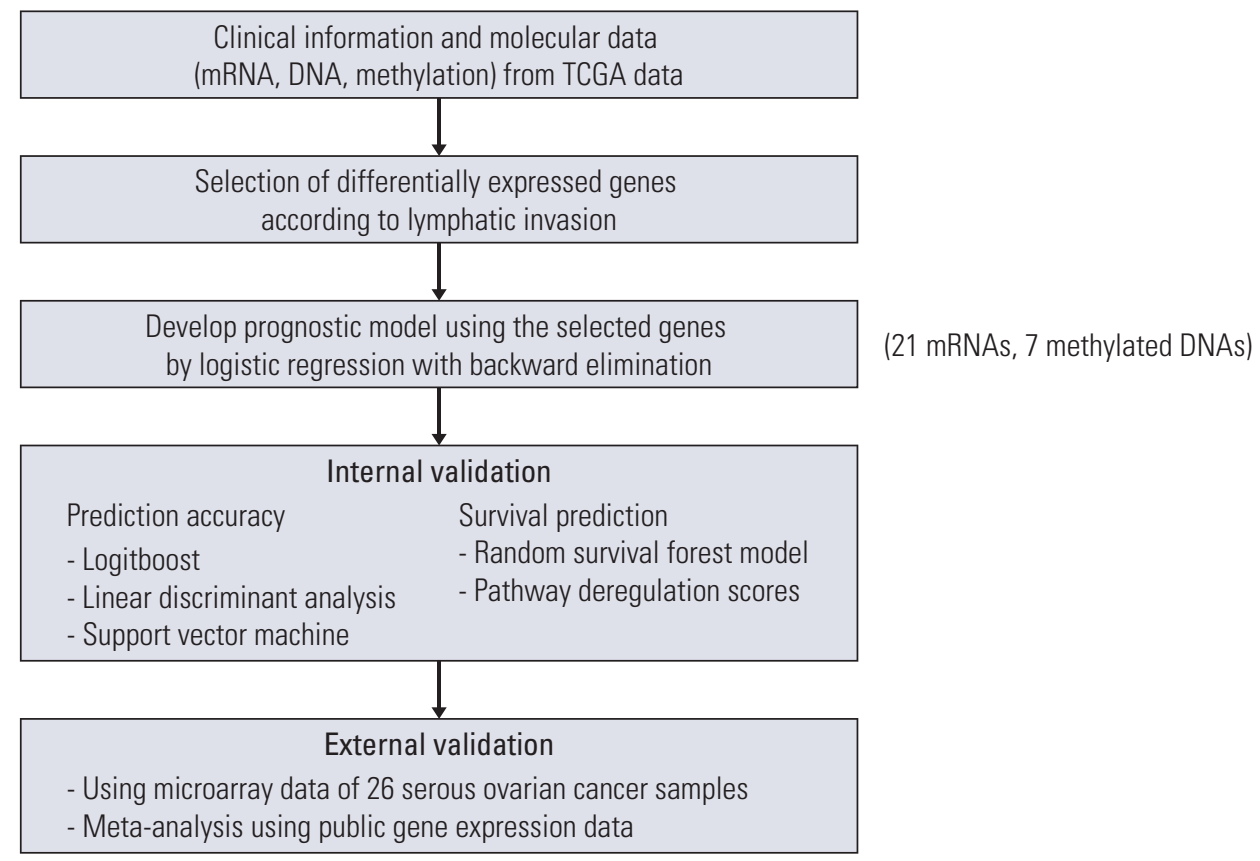

Fig. 1. Flowchart of analysis. TCGA, The Cancer Genome Atlas.

\section{Results}

\section{Identification of molecular signatures associated with lymphatic invasion}

Flowchart illustrating analysis is shown in Fig. 1. Analysis of gene expression profiles with or without lymphatic invasion identified several differentially expressed mRNAs ( $\mathrm{n}=21)$, and methylations $(\mathrm{n}=7)$, which were used for subsequent analysis (S1 and S2 Tables). Of these, six mRNAs were overexpressed, and 15 mRNAs were downregulated. For methylation, two genes were overexpressed, and five genes were downregulated.

\section{Validation of lymphatic invasion prediction using molec- ular signatures}

The clustering of samples using selected genes correlated well with lymphatic invasion status (Fig. 2). We performed ROC analyses to extract the performance of the prediction model. The average AUC value supports good performance of the model. The AUC values for the mRNA, and methylation models were 0.952 and 0.688 , respectively (Fig. $3 \mathrm{~A}$ and B). The gene expression data showed the best performance for the AUC of mRNA data compared with the other data sources (methylation data). For predicting lymphatic invasion, logistic regression, LDA, and SVM algorithm showed a high C-index in mRNA and methylation (Fig. 3C, E, and F).

\section{Prediction analysis of PFS and OS with molecular signa- tures}

To test whether genomic data can supply more prognostic power by combining with clinical factors, we created a RSF model by incorporating the clinical factors with each type of molecular signatures. Using a RSF model, our data showed that genomic data predicted PFS better than clinical variables, and the incorporated models showed significantly better predictive power for PFS compared to the clinical variable-only models (Fig. 4A, $\mathrm{p}<0.001$ and Fig. 4B, $\mathrm{p}=0.008$ ). However, gene signature did not predict $O S$ better than clinical variable model (Fig. 4C and D).

In addition, the Pathifier algorithm (PDS) divided patients into a high-risk and a low-risk group, which resulted in a statistically significant difference in OS with data for only mRNA ( $p=0.011$ ) (Fig. 5). The survival of patients according to lymphatic invasion did not show a statistically significant association with OS. However, patients with a higher PDS showed better OS, indicating the prognostic significance of the molecular signature of lymphatic invasion. For PFS, the difference according to PDS score was not significant (data not shown). 


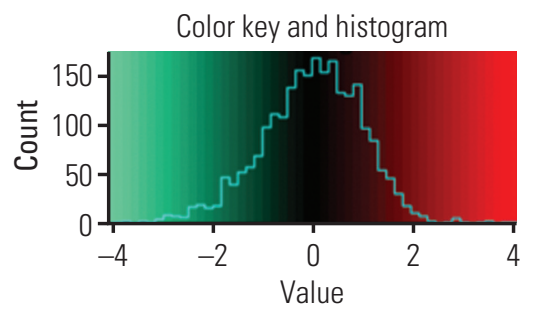

- Lymphatic invasion(-) - Lymphatic invasion(+)

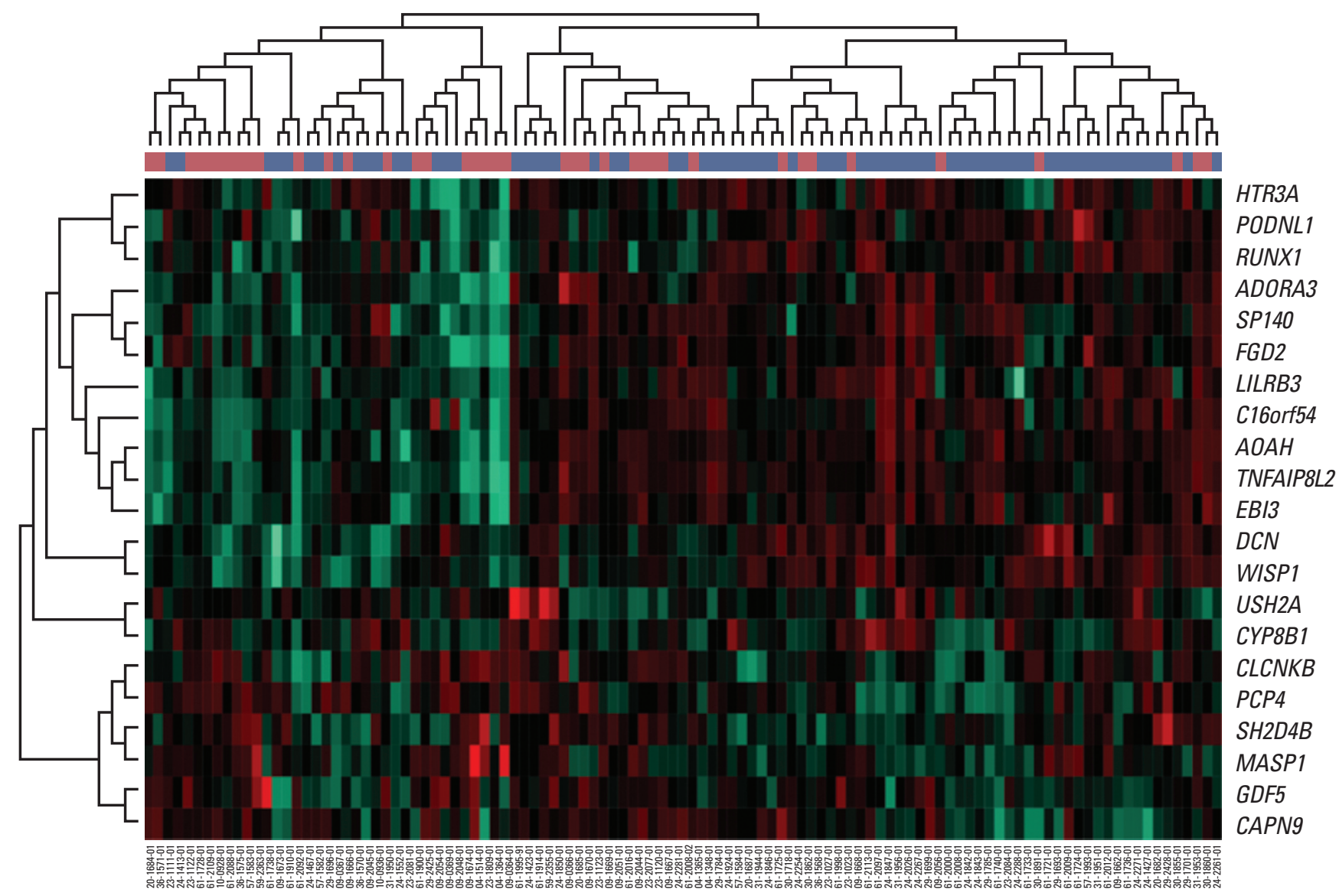

Fig. 2. Expression heat map of mRNAs included in the predictive model. Each column represents one patient and each row represents an mRNA included in the model $(n=21)$, sorted on the basis of the established prognostic index. The plot above the heat map shows the specific risk score index for each sample. In the heatmap, green represents low expression and red represents high expression.

\section{External validation of classifier using microarray data of 26 ovarian cancer samples}

Fig. 6A shows hierarchical clustering of gene expression showing more frequent lymphatic invasion in cluster $1(8 / 10)$ than cluster 2 (7/16). LDA and SVM algorithm showed a high C-index (median of 0.64 and 0.63 , respectively) showing good performance of predicting lymphatic invasion. Additionally, we compared the prediction accuracy of magnetic resonance imaging (MRI) and molecular signature in our patient's cohort (Fig. 6B). Molecular signature alone was inferior to preoperative MRI. However, the combination of MRI and gene signature showed higher prediction of lymphatic invasion.

Using a RSF model, incorporating molecular data and clinical variables improved the prediction of PFS and OS compared to using only clinical variables (age, FIGO stage, grade, residual disease status after primary debulking surgery, and lymphatic invasion) ( $\mathrm{p}=0.005$ and $\mathrm{p}<0.001$, respectively) 
mRNA

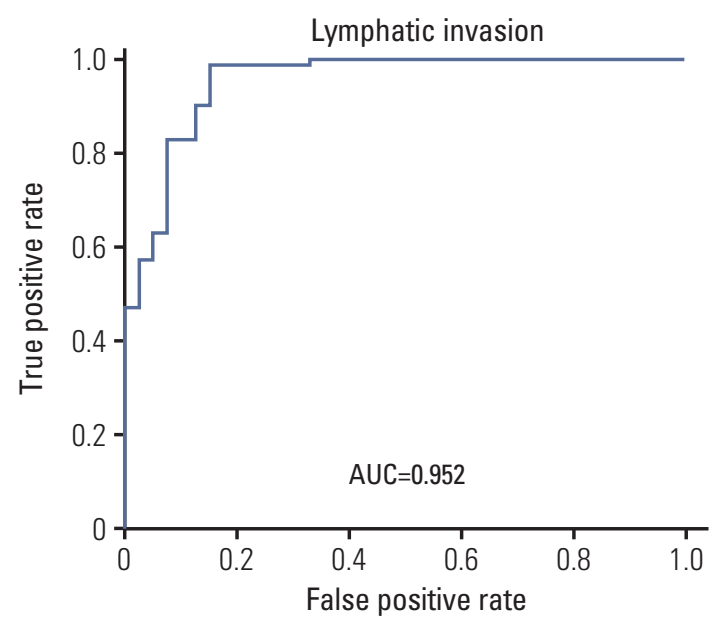

C

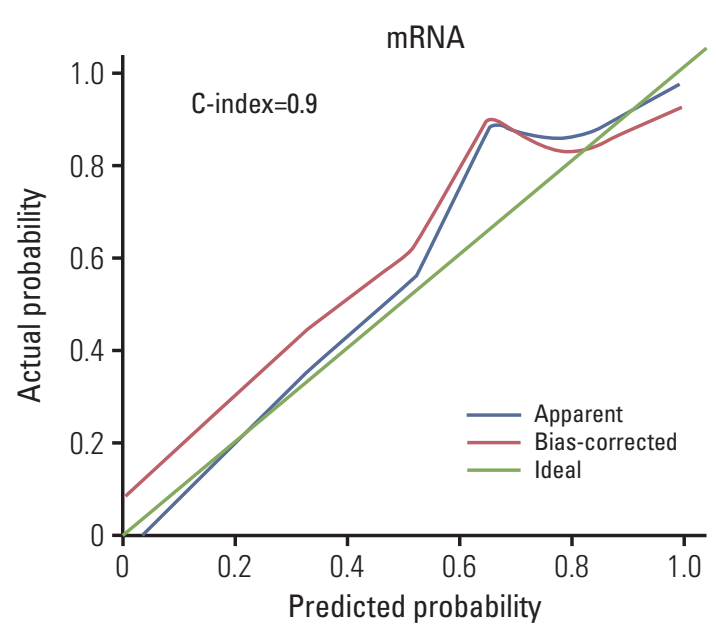

$\mathbf{E}$

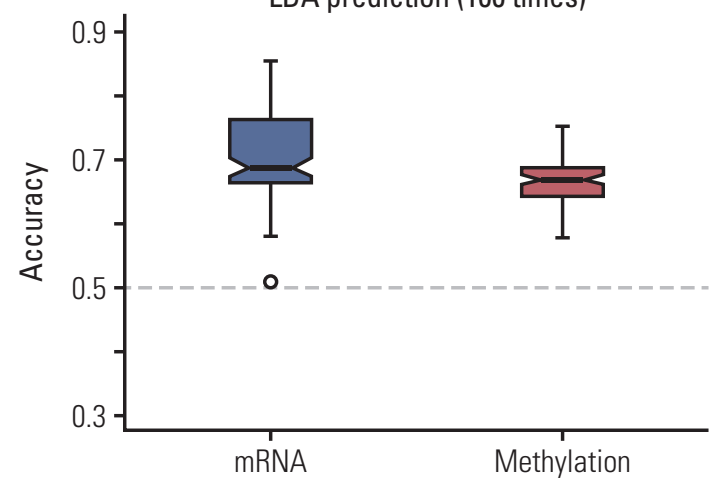

A

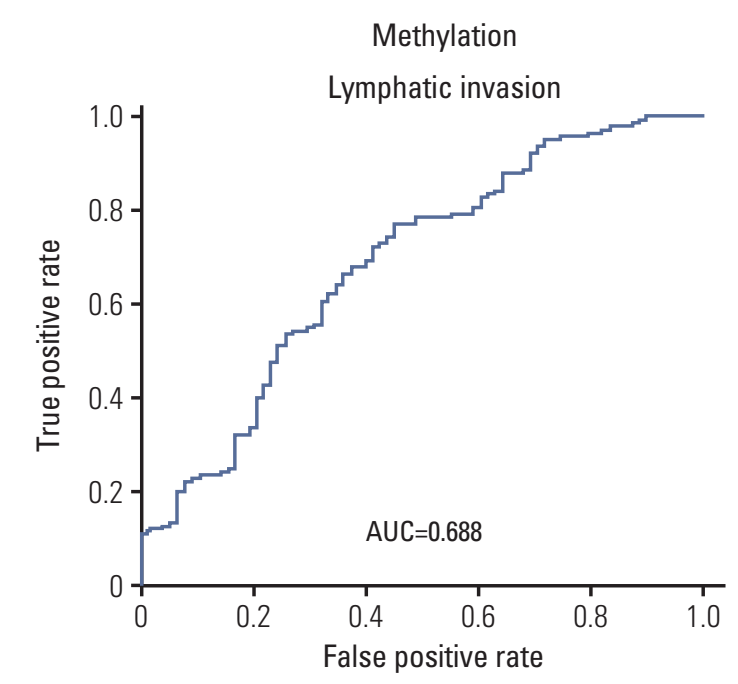

B

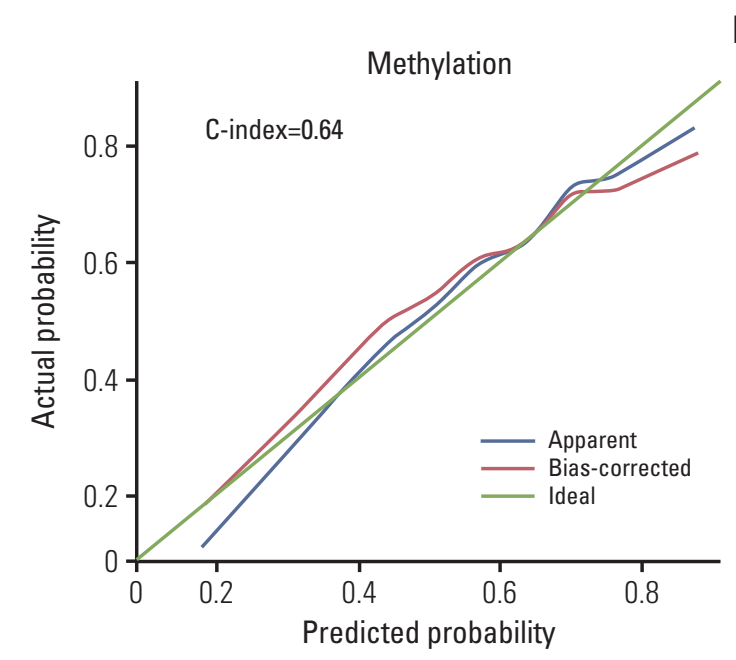

D

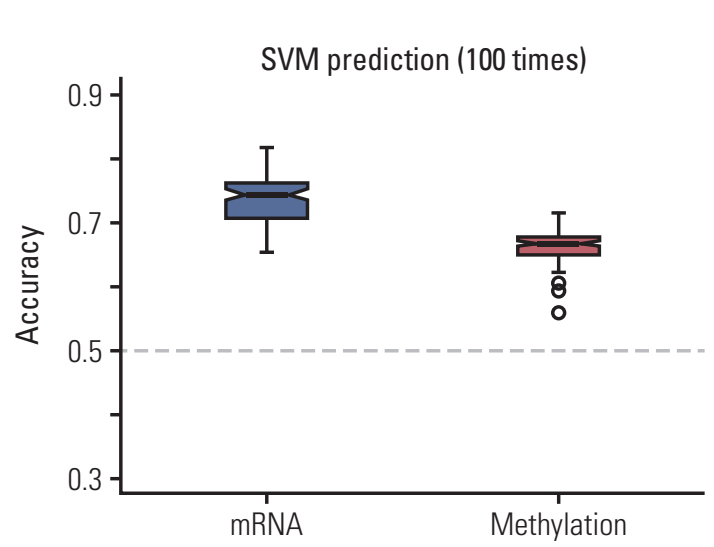

Fig. 3. Accuracy of predicting lymphatic invasion. (A, B) Receiver operating characteristic curve of the prediction of lymphatic invasion using molecular signatures. (C, D) Logistic regression with C-index. (E) Linear discrimination analysis (LDA). (F) Supporter vector machine (SVM). The Y-axis represents the accuracy of the C-index. AUC, area under the curve. 
A
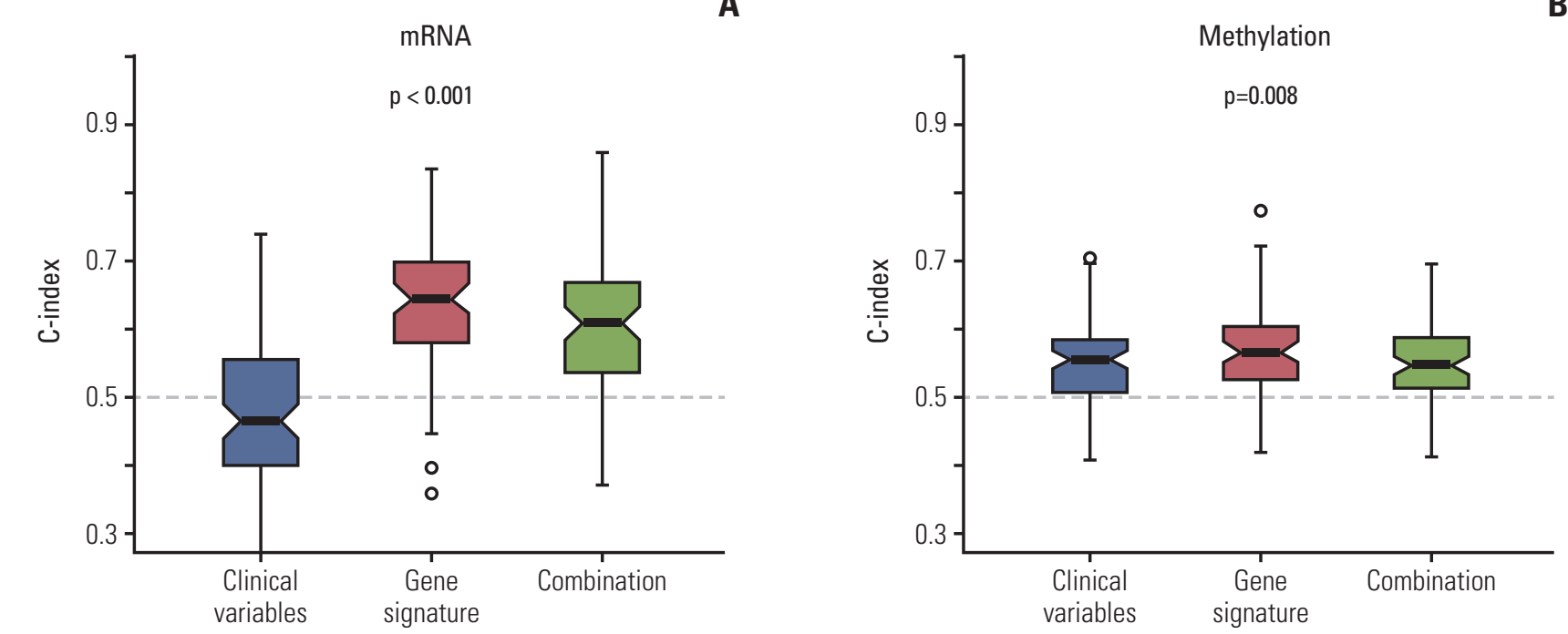

B

B
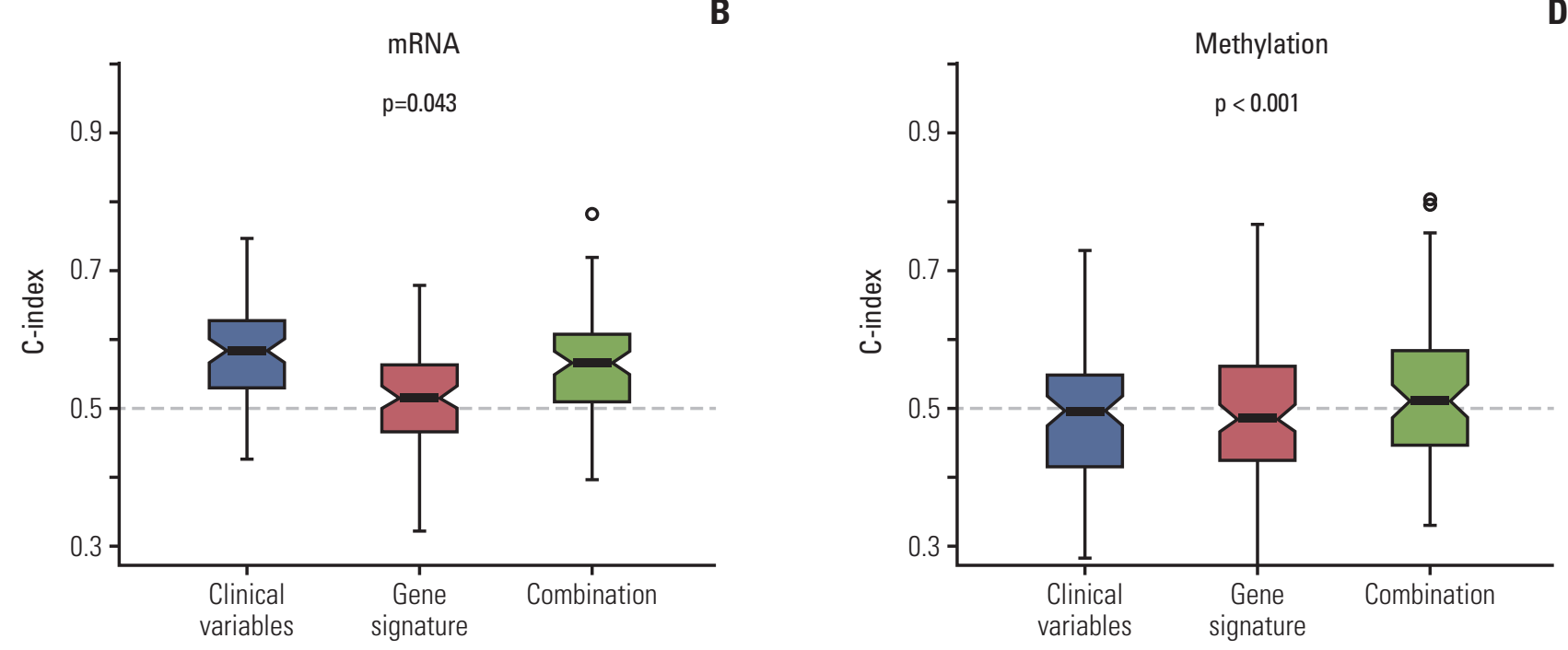

Fig. 4. Random survival forest model for prediction of survival. (A, B) Progression-free survival. (C, D) Overall survival.

(Fig. 6C and D). Meta-analysis of the gene expression data using curatedOvarianData database showed clinical significance of eight genes of 21 selected genes for OS (S3 Table and S4 Fig.).

\section{Discussion}

Until recently, not many gene expression-related molecular markers predicting prognosis had been developed for clinical field, and none of the currently known molecular signatures are generally used in clinical setting for EOC. In this study, we evaluated the prediction of lymphatic invasion and survival based on different level of genetic data types (mRNA and methylated DNA) for potential prognostic relevance and demonstrated the clinical use of large-volume molecular data in EOC. Integrating molecular data statistically increased the model performance compared with the clinical variable-only model.

The considerable effort for molecular characterization of cancer has been made through TCGA [24]. The TCGA research network presented number of papers on the analy- 

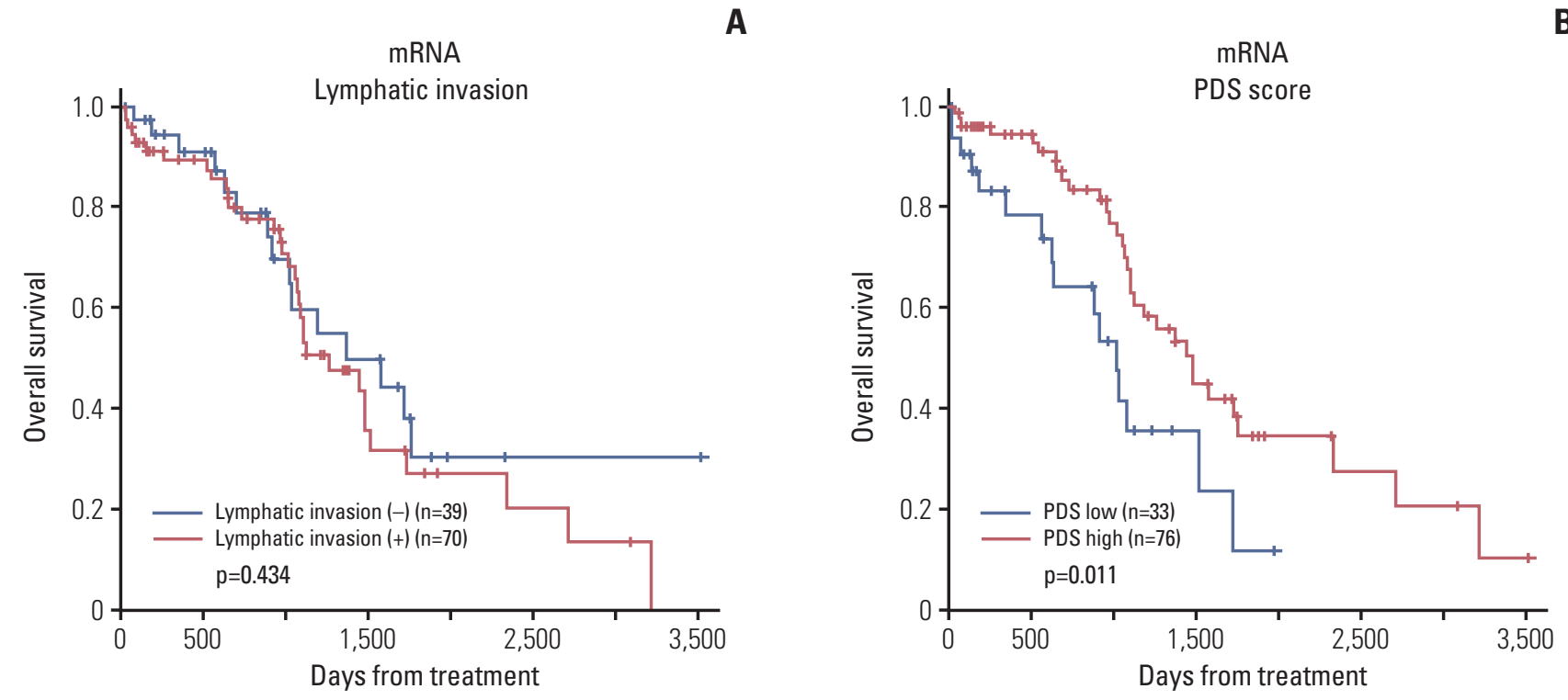

Fig. 5. Kaplan-Meier survival curve according to lymphatic invasion (A) and pathway deregulation score (PDS) (B). The graph according to PDS shows Kaplan-Meier plots of two groups defined by the level of deregulation (high and low) of primary tumor samples. High deregulation scores are associated with better prognosis.

sis of gene expression and DNA methylation data for multiple cancers. By controlling and silencing gene expression through the interaction of methylcytosine binding proteins with other structural components of chromatin, DNA methylation contributes to cancer development [25]. mRNA also has an impact on gene expression and thus may have prognostic significance.

Cancer is the result of various steps in the biological system from genome to proteome, and a single fold of biological information may be insufficient for full explanation of tumor behavior or its mechanisms [14]. In this study, we applied analysis using multiple levels of molecular signatures (DNA methylation and mRNA expression) to the prediction of lymphatic invasion in EOC. The key point in this study is the increase in prognostic prediction by incorporating data from genomic data sources. To the best of our knowledge, this study is the first to assess the multiple molecular-based classifications of lymphatic invasion in EOC patients based on analysis of genomic and clinical features. Predicting lymphatic invasion using molecular classifier will enable physicians to reduce unnecessary invasive procedures and morbidity. In addition, molecular classifier also will be useful to make accurate prediction of prognosis. Possibly, after validation of the molecular classifier, using (easily obtainable) microarray and methylation chip could be applied to the clinical practice.

Lymphatic invasion is considered as a predictor of the aggressiveness of EOC. Interestingly, molecular signatures for lymphatic invasion was not significantly correlated with survival, but model predicting lymphatic invasion showed significant correlation with survival in our study. Possible reason would be addition of clinical variables in multivariate analysis during developing model. Also, degree of lymphatic invasion had been considered to be determinants for OS [8] which was not specifically classified by numbers for present study. In this study, we found that although information about the lymphatic invasion alone was not sufficient, it still can affect the efficiency of prediction using gene expressions.

For additional information, we found model for survival outcome (S5 and S6 Figs.), but the genes selected for survival outcome were different from the genes selected for lymphatic invasion. Also in meta-analysis of the gene expression data using curatedOvarianData database, only eight genes of 21 selected genes were significant. Representatively, RUNX1 is reported to be associated with female-related cancers as well as involved in female sex development and a crucial mediator of female hormone signaling [26]. CCN4 is aberrantly expressed in numerous cancer including ovarian cancer [27], and its transcript levels are lower in node-positive highgrade tumors and in patients with poor clinical outcome for breast cancer [28]. Low CAPN9 is associated with adverse disease-specific survival following endocrine therapy in breast cancer, and detection of ADORA3 was reported as a potential target for antibody-based therapy in p53 mutated 

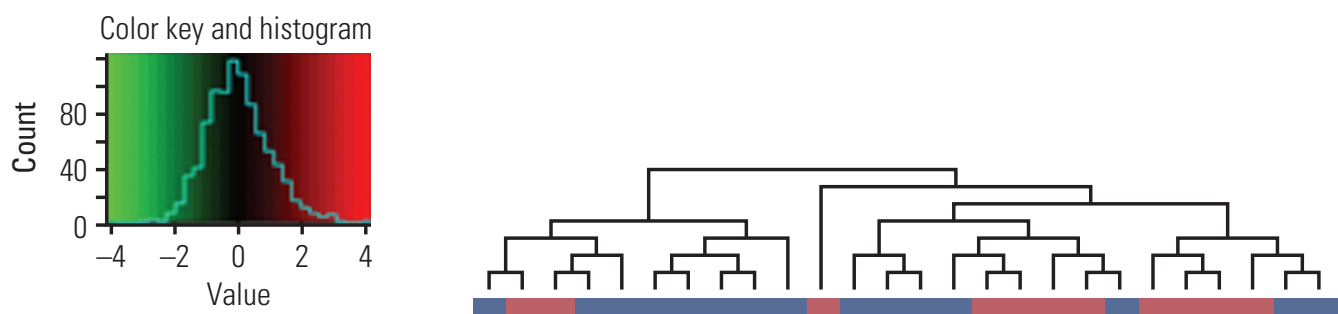

Lymphatic invasion(-)

- Lymphatic invasion(+)

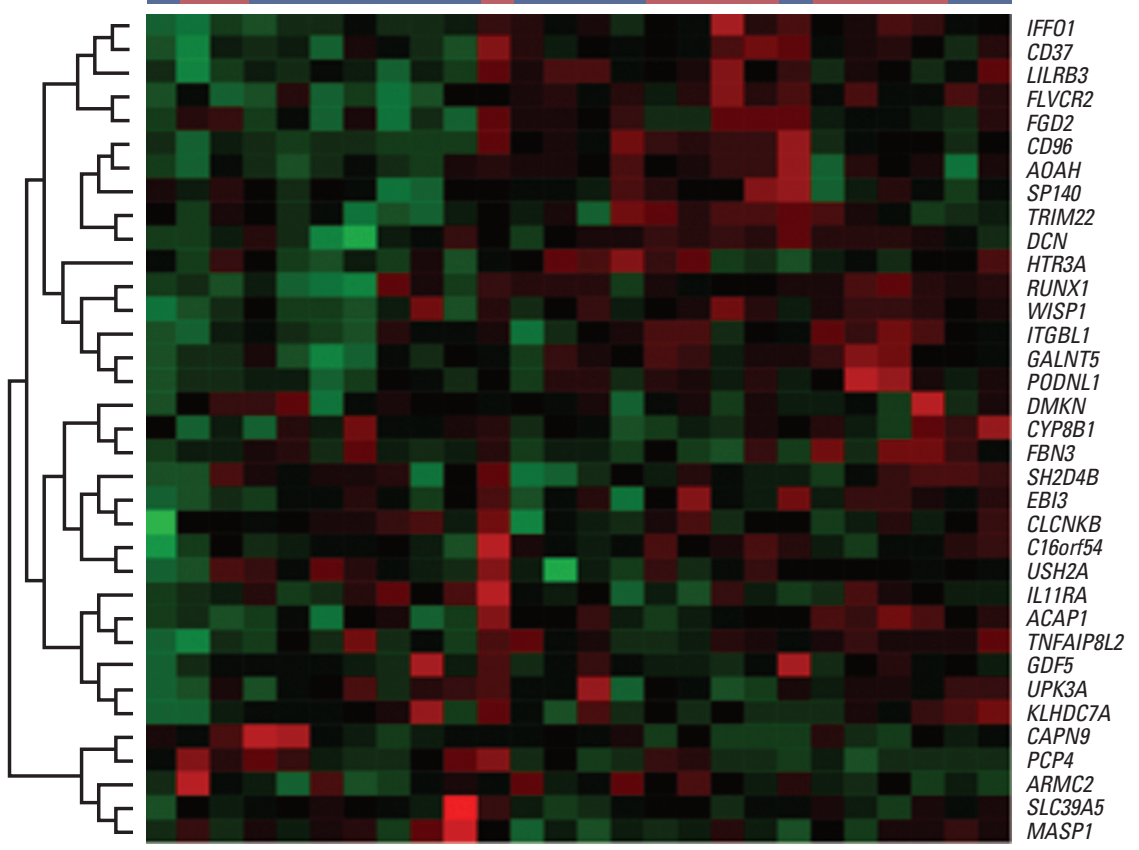

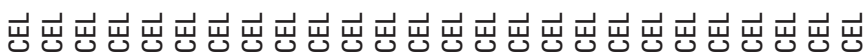
望

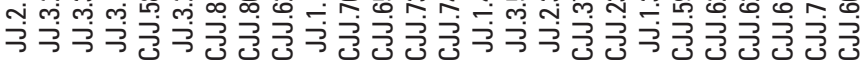

Fig. 6. Validation with microarray data from 26 ovarian cancer samples from Samsung Medical Center. (A) Hierarchical clustering of gene expression according to lymphatic invasion. (Continued to the next page)

tumors [29]. However, as mentioned earlier, molecular signatures for lymphatic invasion was not significantly correlated with survival possibly due to different pathophysiology that influenced the survival of the patients for each genes.

Our study has some limitations even though we offered novel concepts in the translation of molecular data to clinical practice. First, we used only data-mining process to creating prognostic model. We may have skipped some important clinical characteristics that could be distinguished by a previously known candidate gene. Second, engrafting multiple types of molecular data is technically challenging because of the problem with overfitting and extended co-linearity of large-volume biological data. Third, the clinical information from patient samples may not be appropriately accurate compared to information from large-scale clinical trials. Also, there were differences in data analyzing methods between internal and external cohort. For TCGA data analysis, RNAseqV2was used, but for external cohort, microarray (AffymetrixGeneChip Human Gene 1.0 ST oligonucleotide arrays) was used. RNA sequencing is known to provide increased specificity and sensitivity, enhanced detection of differential expression compared to microarray method. Inaccurate comparison may have been made. And lastly, the required time for the coordinated analysis described in this study is important for the clinical applications [30], which is 
B

mRNA

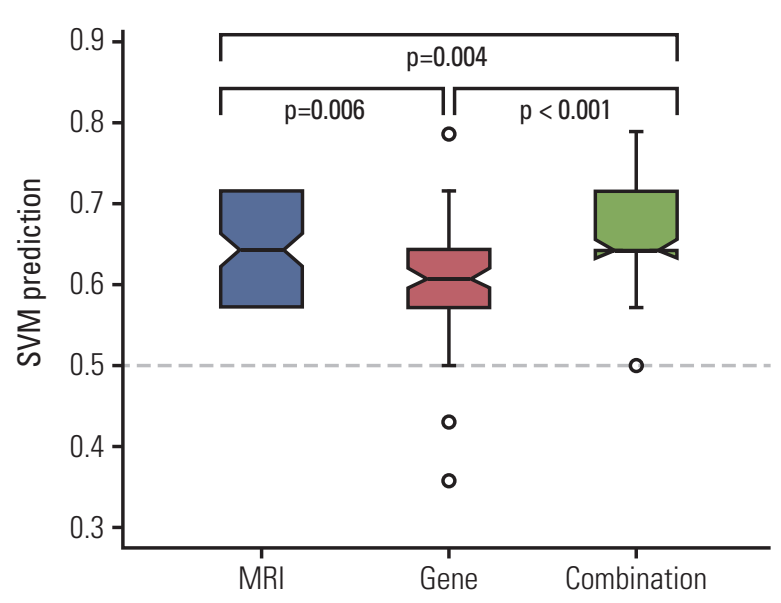

mRNA

Overall survival

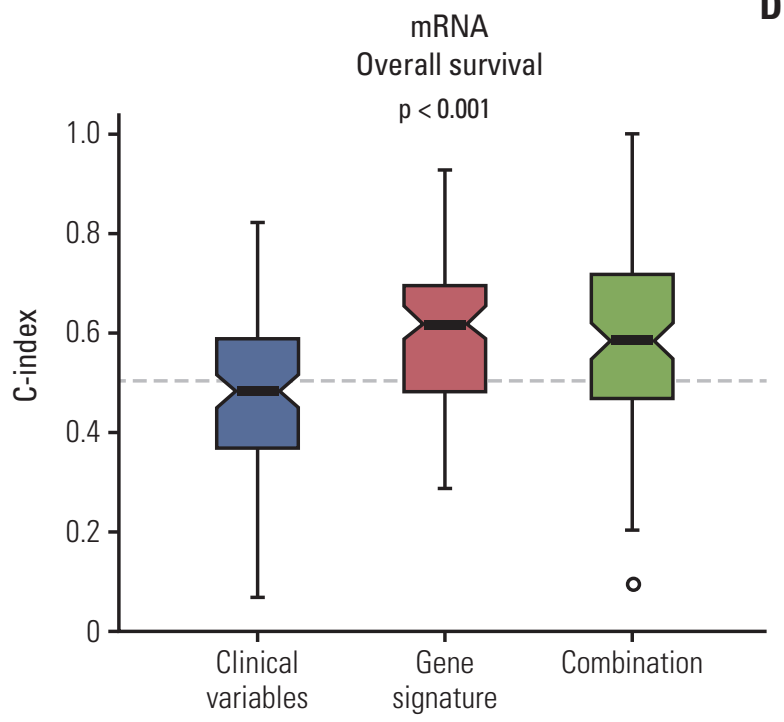

D
Progression-free survival

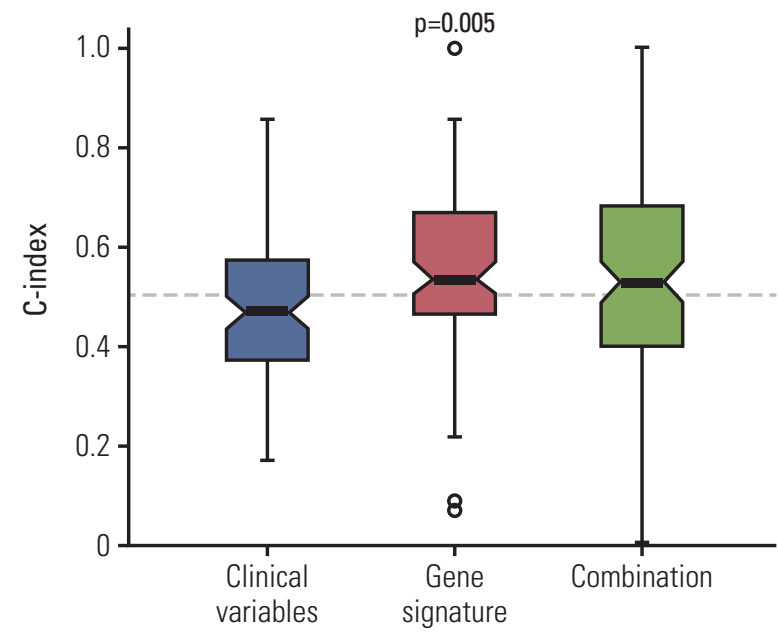

Fig. 6. (Continued from the previous page) (B) Prediction accuracy of magnetic resonance imaging (MRI), molecular signature, and combination. (C, D) Random survival forest model showing incorporating molecular data and clinical variables improved the prediction of progression-free survival and overall survival.

expected evolve in near future. Thus, further investigations, such as external validations with large-scale clinical triallevel follow-up, are needed to validate our results.

In conclusion, our study showed that molecular signatures (mRNA and methylated DNA) based on lymphatic invasion improved prognostic stratification for EOC patients. Incorporating molecular data statistically increased the predictability compared with the model based only on the clinical variable. The signatures require further investigation to generate clinical-level prognostic analysis.

\section{Electronic Supplementary Material}

Supplementary materials are available at Cancer Research and Treatment website (http:// www.e-crt.org).

\section{Conflicts of Interest}

Conflict of interest relevant to this article was not reported. 


\section{Acknowledgments}

This research was supported by Basic Science Research Program through the National Research Foundation of Korea (NRF) funded by the Ministry of Education (2013R1A1A-2013629), a grant of the
Korea Health Technology R\&D Project through the Korea Health Industry Development Institute(KHIDI), funded by the Ministry of Health \& Welfare, Republic of Korea (HI14C3418), and grant from the National R\&D Program for Cancer Control, Ministry for Health, Welfare and Family affairs, Republic of Korea (0920010).

\section{References}

1. Siegel RL, Miller KD, Jemal A. Cancer statistics, 2015. CA Cancer J Clin. 2015;65:5-29.

2. Lim MC, Moon EK, Shin A, Jung KW, Won YJ, Seo SS, et al. Incidence of cervical, endometrial, and ovarian cancer in Korea, 1999-2010. J Gynecol Oncol. 2013;24:298-302.

3. Jayson GC, Kohn EC, Kitchener HC, Ledermann JA. Ovarian cancer. Lancet. 2014;384:1376-88.

4. Ahluwalia A, Yan P, Hurteau JA, Bigsby RM, Jung SH, Huang $\mathrm{TH}$, et al. DNA methylation and ovarian cancer. I. Analysis of CpG island hypermethylation in human ovarian cancer using differential methylation hybridization. Gynecol Oncol. 2001; $82: 261-8$

5. Cannistra SA. Cancer of the ovary. N Engl J Med. 2004;351: 2519-29.

6. Rubin SC, Randall TC, Armstrong KA, Chi DS, Hoskins WJ. Ten-year follow-up of ovarian cancer patients after secondlook laparotomy with negative findings. Obstet Gynecol. 1999;93:21-4.

7. Vaidya AP, Curtin JP. The follow-up of ovarian cancer. Semin Oncol. 2003;30:401-12.

8. Matsuo K, Sheridan TB, Yoshino K, Miyake T, Hew KE, Im $\mathrm{DD}$, et al. Significance of lymphovascular space invasion in epithelial ovarian cancer. Cancer Med. 2012;1:156-64.

9. Lim MC, Lee JS, Nam BH, Seo SS, Kang S, Park SY. Lower extremity edema in patients with early ovarian cancer. J Ovarian Res. 2014;7:28.

10. Kim SI, Lim MC, Lee JS, Lee Y, Park K, Joo J, et al. Impact of lower limb lymphedema on quality of life in gynecologic cancer survivors after pelvic lymph node dissection. Eur J Obstet Gynecol Reprod Biol. 2015;192:31-6.

11. Swanton C, Caldas C. Molecular classification of solid tumours: towards pathway-driven therapeutics. Br J Cancer. 2009;100:1517-22.

12. Cardin V, Friston KJ, Zeki S. Top-down modulations in the visual form pathway revealed with dynamic causal modeling. Cereb Cortex. 2011;21:550-62.

13. Chin L, Gray JW. Translating insights from the cancer genome into clinical practice. Nature. 2008;452:553-63.

14. Hanash S. Integrated global profiling of cancer. Nat Rev Cancer. 2004;4:638-44.

15. Dettling M, Buhlmann P. Boosting for tumor classification with gene expression data. Bioinformatics. 2003;19:1061-9.

16. Vapnik VN. An overview of statistical learning theory. IEEE
Trans Neural Netw. 1999;10:988-99.

17. Furey TS, Cristianini N, Duffy N, Bednarski DW, Schummer M, Haussler D. Support vector machine classification and validation of cancer tissue samples using microarray expression data. Bioinformatics. 2000;16:906-14.

18. Ishwaran H, Kogalur UB. Consistency of random survival forests. Stat Probab Lett. 2010;80:1056-64.

19. Drier Y, Sheffer M, Domany E. Pathway-based personalized analysis of cancer. Proc Natl Acad Sci U S A. 2013;110:6388-93.

20. Hastie T, Stuetzle W. Principal curves. J Am Stat Assoc. 1989;84:502-16.

21. Choi CH, Choi JJ, Park YA, Lee YY, Song SY, Sung CO, et al. Identification of differentially expressed genes according to chemosensitivity in advanced ovarian serous adenocarcinomas: expression of GRIA2 predicts better survival. Br J Cancer. 2012;107:91-9.

22. Ganzfried BF, Riester M, Haibe-Kains B, Risch T, Tyekucheva $S$, Jazic I, et al. curatedOvarianData: clinically annotated data for the ovarian cancer transcriptome. Database (Oxford). 2013;2013:bat013.

23. Harrell FE Jr, Lee KL, Mark DB. Multivariable prognostic models: issues in developing models, evaluating assumptions and adequacy, and measuring and reducing errors. Stat Med. 1996;15:361-87.

24. Cancer Genome Atlas Research Network. Comprehensive genomic characterization defines human glio-blastoma genes and core pathways. Nature. 2008;455:1061-8.

25. Bibikova M, Lin Z, Zhou L, Chudin E, Garcia EW, Wu B, et al. High-throughput DNA methylation profiling using universal bead arrays. Genome Res. 2006;16:383-93.

26. Riggio AI, Blyth K. The enigmatic role of RUNX1 in femalerelated cancers: current knowledge \& future perspectives. FEBS J. 2017;284:2345-62.

27. Gurbuz I, Chiquet-Ehrismann R. CCN4/WISP1 (WNT1 inducible signaling pathway protein 1 ): a focus on its role in cancer. Int J Biochem Cell Biol. 2015;62:142-6.

28. Davies SR, Watkins G, Mansel RE, Jiang WG. Differential expression and prognostic implications of the CCN family members WISP-1, WISP-2, and WISP-3 in human breast cancer. Ann Surg Oncol. 2007;14:1909-18.

29. Riccardo F, Arigoni M, Buson G, Zago E, Iezzi M, Longo D, et al. Characterization of a genetic mouse model of lung cancer: a promise to identify non-small cell lung cancer therapeutic 
targets and biomarkers. BMC Genomics. 2014;15 Suppl 3:S1.

30. Feng N, Ching T, Wang Y, Liu B, Lin H, Shi O, et al. Analysis of microarraydata on gene expression and methylation to identify long non-coding RNAs in non-small cell lung cancer. Sci Rep. 2016;6:37233. 